\title{
Plasmonic metasurface for efficient ultrashort pulse laser-driven particle acceleration
}

\author{
Doron Bar-Lev and Jacob Scheuer" \\ School of Electrical Engineering, Tel Aviv University, Ramat Aviv, Tel-Aviv 69978, Israel \\ Center for Nano-Science and Nanotechnology, Tel Aviv University, Ramat Aviv, Tel-Aviv 69978, Israel
}

(Received 18 February 2014; published 15 December 2014)

\begin{abstract}
A laser-driven particle accelerator based on plasmonic nanoantennas is proposed and analyzed. The concept utilizes the enhancement and localization of the electric field by nanoantennas to maximize the acceleration gradient and to overcome potential metallic losses. The structure is optimized for accelerating relativistic particles using a femtosecond laser source operating at $800 \mathrm{~nm}$ and is shown to support the bandwidth of ultrashort laser pulses (up to $16 \mathrm{fs}$ ) while providing a high acceleration gradient potentially reaching $11.6 \mathrm{GV} / \mathrm{m}$.
\end{abstract}

DOI: 10.1103/PhysRevSTAB.17.121302

PACS numbers: 41.75.Jv, 41.75.Lx, 73.20.Mf, 78.67.-n

\section{INTRODUCTION}

Laser-driven particle accelerators constitute a major step towards compact and cost-effective devices for high-energy particle beam generation and, thus, hold promise for a variety of applications for high-energy physics, radiation generation, medicine, and more. These devices utilize the high electric field of a laser beam to accelerate charged particles, where in order to attain net acceleration the oscillations of the longitudinal electric field and the position of the particles beam must be synchronized [1]. Because of its transverse nature, the electric field of a laser pulse copropagating with the particles cannot be utilized for their acceleration [2]. Consequently, several techniques have been suggested to rotate the electric field and gain a synchronized longitudinal component that can be used [2-12]. Nonetheless, natural group velocity dispersion, relatively large mode volume, and many-cycle fieldbuildup times render these schemes less efficient for ultrashort pulse laser utilization [13]. As such lasers are characterized by a very large electric field, exploiting them can be highly efficient. A different approach, which was recently demonstrated experimentally, utilizes a single-pass dielectric laser accelerator based on periodic field reversal $[13,14]$. The nonresonant nature of this structure supports ultrashort pulse operation. Nevertheless, because of its relatively low $Q$, the attained average longitudinal field (acceleration gradient) in a quartz-based structure is $G=0.49 E_{0}$, where $E_{0}$ is the electric field amplitude of the input plane wave. A principal question to be asked is whether it is possible to design a laser accelerator which

\footnotetext{
*kobys@eng.tau.ac.il

Published by the American Physical Society under the terms of the Creative Commons Attribution 3.0 License. Further distribution of this work must maintain attribution to the author(s) and the published article's title, journal citation, and DOI.
}

provides a larger acceleration gradient while supporting ultrashort laser operation.

One of the common conceptions in laser accelerator design is that losses at optical wavelengths render metallicbased devices inefficient [2,12]. However, a metallic structure exhibiting a plasmonic resonance leads to field enhancement in the near-field zone of the structure [15] which can be utilized for compensating these losses and attaining a substantial acceleration gradient. Nanoantennas are nanometer-scale metallic objects which interact with electromagnetic (EM) waves and can be designed to exhibit localized plasmon resonances at optical and infrared frequencies. These nanostructures were shown to enhance the efficiency of numerous optical phenomena such as photodetection, photovoltaics, photogeneration, nonlinear optical phenomena, imaging, and more [16]. A nanoantenna can concentrate the EM energy into an ultrasmall volume [17], resulting in a large enhancement of the local field [18-21] which is attributed to the plasmonic resonances [22]. Consequently, power enhancement factors exceeding $40 \mathrm{~dB}$ have been demonstrated [23]. Moreover, close to resonance, plasmonic modes exhibit strong coupling to radiating modes which renders the impact of metallic losses less significant [24]. Similar to their conventional rf counterparts, the resonances of nanoantennas are related to their dimensions [25]. In addition, when considering nanoantenna arrays, the coupling between adjacent elements [26], the structure surroundings [27,28], and the array properties [29] can substantially affect the field enhancement. Thus, the amplitude, phase, and polarization of the field in the vicinity of plasmonic metasurfaces comprising nanoantennas can be controlled as desired [30,31]. Here, we show that plasmonic metasurfaces can be applied for efficient laser acceleration suitable for ultrashort laser operation. In the proposed metasurface laser accelerator (MLA), the electric field is enhanced by an array of metallic nanoantennas and is directed towards the 
vacuum channel (particles channel), thus enhancing the acceleration gradient. In addition, the structure is designed such that the field is mostly localized in the vacuum and it yields a specific spatial phase profile of the field which enhances the gradient further. Consequently, a large acceleration gradient bound is attained despite the use of metals. The proposed structure is designed for accelerating relativistic electrons (i.e., $\beta \approx 1$ ). Nevertheless, nanoantenna array versatility enables the design of MLAs which are optimized for slower particle beams as well.

The rest of the paper is organized as follows: Section II describes the proposed MLA structure and presents the accelerating field distribution and the resulting acceleration gradient. The dependency of this acceleration gradient on specific design parameters as the vacuum channel width and the Au layer thickness is discussed in Sec. III. In Sec. IV, the expected damage threshold of the structure is analyzed and the corresponding maximal unloaded acceleration gradient bound is deduced. Following that, the loaded gradient and bunch charge limit are also calculated. In Sec. V, we address the ultrashort laser operation and infer the shortest supported pulse. Finally, in Sec. VI, we summarize the main findings and conclude.

\section{MLA STRUCTURE AND ACCELERATING FIELD DISTRIBUTION}

Attaining effective acceleration requires the synchronization of the particle beam with the electric field component in the direction of the particle motion (denoted here as the $y$ direction). Consequently, for $\beta \approx 1$ electron beams it is essential that the spatial distribution of the field includes a spatial harmonic with a wave number equal to $k_{0}=2 \pi / \lambda_{0}$, where $\lambda_{0}$ is the center wavelength [2]. A possible method to satisfy this requirement is to introduce a periodic structure with a longitudinal period of $d_{y}=\lambda_{0}$. Let us assume a slablike structure which encloses the particle channel and is symmetric along the $z$ axis. The structure is illuminated symmetrically by two incoming fields which propagate in opposite directions along the $z$ axis and are polarized along the particle channel (see Fig. 1). The electric field in the channel can be expressed in the Floquet form $E_{y}=E_{0}\left(e^{i(k z-\omega t)}+e^{-i(k z+\omega t)}\right) \cdot U(y)$, where $E_{0}$ is the amplitude of each one of the impinging fields and $U(y)$ is a periodic function $U\left(y+\lambda_{0}\right)=U(y)$. Consequently, it is possible to expand the field by its Fourier series:

$$
E(y, z, t)=E_{0}\left(e^{i(k z-w t)}+e^{-i(k z+w t)}\right) \sum_{n} a_{n} e^{i \frac{2 \pi n}{\lambda} n y} .
$$

By assuming that the electrons are traveling along $z=0$ and applying the propagation relation $t \approx y / c$, the field along the trajectory can be expressed as a function of $y$ only:

$$
E(y)=2 E_{0} e^{-i \frac{2 \pi}{\pi_{0}} y} \sum_{n} a_{n} e^{i \frac{2 \pi}{\pi_{0}} n y} .
$$

The acceleration gradient $G$ is defined as the average field along a single period [13], yielding

$$
G=\frac{2 E_{0}}{\lambda_{0}} \sum_{n} \int_{0}^{\lambda_{0}} a_{n} e^{i \frac{2 \pi}{2} y(n-1)} d y=2 E_{0} a_{1} .
$$

As can be seen, the acceleration gradient stems from the fundamental spatial harmonic of the field. Nevertheless, due to the field enhancement induced by the nanoantennas, the component $a_{1}$ can be large, as the field is focused from a unit cell area to a localized point. To enhance the fundamental harmonic while retaining a relatively high field enhancement, we propose utilizing a plasmonic metasurface which consists of a periodic arrangement of unit cells comprising two Au nanoantennas: a slot and a patch [see Fig. 1(a)]. As these nanoantennas are complementary in the sense that patches excite electric currents while slots excite "magnetic currents", one can expect that combining them into a unit cell may yield the desired electric field phase profile for effective acceleration. Clearly, the electron beam might be affected by the existence of transverse electric fields. Therefore, to maximize the acceleration and maintain electron beam quality [2], we utilize a symmetric MLA structure comprising two parallel metasurfaces of thickness $t$ which are patterned on $\mathrm{SiO}_{2}$ substrates. These metasurfaces are placed at the opposite facets of a $d_{z}$-wide vacuum channel as depicted in Fig. 1(b). The acceleration gradient upper bound stems from the expected damage threshold of the MLA structure. At the nanoantenna resonance, the field is expected to be localized in the vicinities of the gaps of the structure. Therefore, to minimize the field in the $\mathrm{SiO}_{2}$ substrates and attain a large structural damage threshold, we remove the $\mathrm{SiO}_{2}$ from the vicinity of the metallic-gap boundaries, creating $80 \mathrm{~nm}$ deep mushroomlike vacancies beneath the slots and the gaps adjacent to the patches [see highlighted oval areas in Figs. 1(b) and 1(c)]. Such apertures can be realized by introducing an etching stage to the MLA

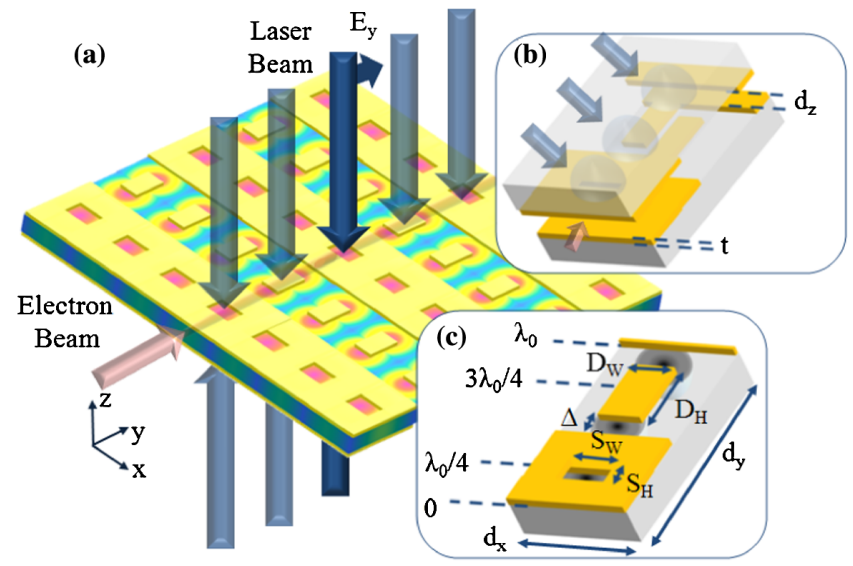

FIG. 1. MLA schematic description. (a) top view; (b) unit cell structure; (c) unit cell dimensions. 
fabrication process. The structure is periodic in both the $x$ and $y$ directions. The periodicity in $x$ affects the coupling between adjacent unit cells and is optimized for obtaining (approximately) a $\pi$ phase shift in the accelerating field profile after the accelerated electron beam propagates half a unit cell, thus maximizing the acceleration gradient. The periodicity in $y$ is set to be $\lambda_{0}$, therefore maintaining the required phase synchronicity over many MLA periods.

Using a finite difference time domain simulation tool [32], we optimized the MLA dimensions such that it resonates at $\lambda_{0}=800 \mathrm{~nm}$. This choice stems from the availability of high-quality, high-power laser sources at this wavelength (e.g., Ti:sapphire lasers). Nevertheless, different resonances can be designed as well. The impinging laser fields are linearly polarized along the $y$ axis, corresponding to the maximal response of the nanoantennas. In addition, symmetric illumination in the $\pm z$ directions is employed to eliminate the impact of transverse electric fields. Consequently, we normalize the results by $2 E_{0}$, where $E_{0}$ is the amplitude of each one of the impinging fields. The analysis takes into account the measured dispersion of the employed materials as well as the optical losses in the metallic layers [33]. Particularly at $800 \mathrm{~nm}$ the $\mathrm{Au}$ dielectric constant is complex, $\varepsilon=-25.43+1.83 i$, thus resulting in a corresponding skin depth of $25.23 \mathrm{~nm}$. Table I details the MLA dimensions for attaining a resonant response at $800 \mathrm{~nm}$. As can be seen, the array spacing along the $x$ direction, $d_{x}$, is of subwavelength scale; hence, no diffraction lobes are expected in this direction. The $y$ spacing, on the other hand, corresponds to the limit of the $1^{\text {st }}$ diffraction order condition in the vacuum. However, because of the $\mathrm{SiO}_{2}$ substrates, the MLA structure does not suffer from losses that originate from Wood's anomalies. The remainder of the dimensions is on the order of $100 \mathrm{~nm}$, thus feasible by means of contemporary fabrication techniques (e.g. $E$-beam lithography). Figure 2 depicts the distribution of the longitudinal electric field $\left(E_{y}\right)$ along the symmetry axis of the MLA $(x=z=0)$. This axis corresponds to the electron beam trajectory [highlighted line in Fig. 1(a)]. Figure 2(a) depicts the dependence of the $E_{y}$ amplitude on the laser wavelength and denotes the optimal acceleration field at $800 \mathrm{~nm}$ (normalized to $2 E_{0}$ ) as experienced by a relativistic particle with the maximally accelerating phase. Figures 2(b) and 2(c), respectively, depict $E_{y}$ amplitude and phase at $\lambda=800 \mathrm{~nm}$. Clearly, the maximal field is obtained around $\lambda=800 \mathrm{~nm}$ corresponding to the MLA resonance. The field is localized at three different regions in which the normalized amplitude

TABLE I. MLA dimensions [nanometers] for $800 \mathrm{~nm}$ resonance.

\begin{tabular}{lcccccccc}
\hline \hline$d_{x}$ & $d_{y}$ & $d_{z}$ & $t$ & $S_{w}$ & $S_{H}$ & $D_{W}$ & $D_{H}$ & $\Delta$ \\
\hline 500 & 800 & 100 & 40 & 200 & 100 & 150 & 147 & 75 \\
\hline \hline
\end{tabular}

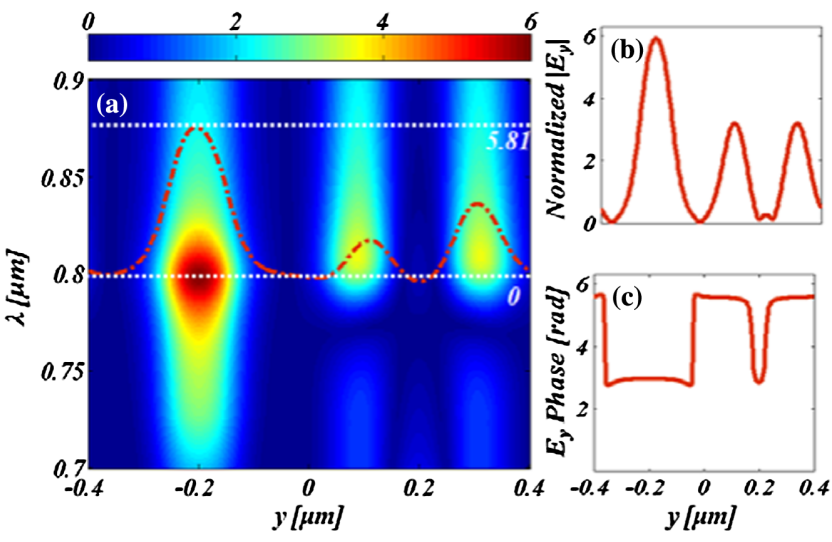

FIG. 2. Longitudinal electric field distribution. (a) Normalized amplitude wavelength dependency; dashed line, optimal acceleration field at $800 \mathrm{~nm}$ as experienced by a relativistic particle with the maximally accelerating phase. (b) Normalized amplitude at $800 \mathrm{~nm}$. (c) Phase at $800 \mathrm{~nm}$.

$\left(\left|E_{y}\right| / 2 E_{0}\right)$ is enhanced by a factor ranging between 6 and 3 [see Fig. 2(b)]. The first region corresponds to the area between the slots openings $\left(y=-\lambda_{0} / 4=-0.2 \mu \mathrm{m}\right)$, while the other two are located symmetrically around $y=\lambda_{0} / 4=0.2 \mu \mathrm{m}$, i.e., at the apertures adjacent to the patches. The phase difference between the first and the two other peaks is $0.83 \pi$ [see Fig. 2(c)], resulting primarily in an acceleration buildup along the electron beam trajectory. Note that there is also a small peak at $y=0.2 \mu \mathrm{m}$ with approximately the same phase as that of the slot antenna peak. This peak lowers the acceleration gradient; however, it is substantially smaller than the other peaks and, therefore, has low impact. The acceleration gradient is evaluated by using Eq. (3), yielding a normalized gradient of $G / 2 E_{0}=1.32$ for the structure parameters in Table I.

Note that, at a given laser beam power, splitting the beam for symmetrical illumination decreases the amplitude of the electric field from each side by a factor of $\sqrt{2}$; however, it increases the acceleration gradient by the same factor. Hence, symmetrical illumination yields a more efficient acceleration. Therefore, when comparing the acceleration gradient to that provided by the dielectric field reversal structure presented in Ref. [13], the MLA attains approximately a 3.81 times larger gradient.

\section{VACUUM CHANNEL WIDTH AND AU LAYER THICKNESS OPTIMIZATION}

The MLA parameters affect the resonance wavelength, the field enhancement, and the electric field phase distribution along the vacuum channel. Consequently, each of these parameters affects the acceleration gradient. Figure 3 shows the impact of the electron channel width $d_{z}$ [Fig. 3(a)] and the Au layer thickness $t$ [Fig. 3(b)] on the normalized acceleration gradient. The gradient increases monotonically for smaller $d_{z}$, reaching a gradient of $3.35 E_{0}$ 

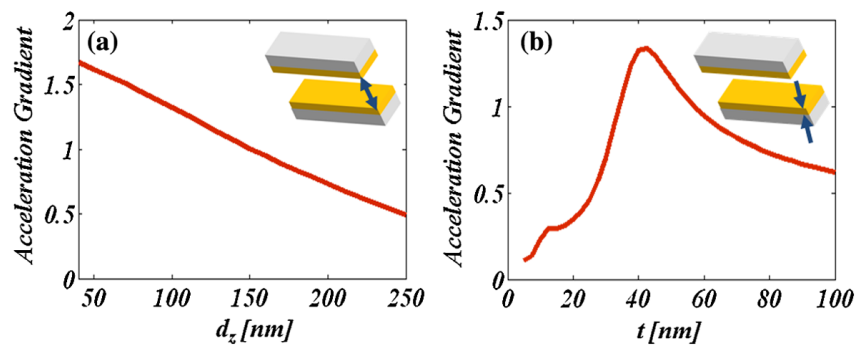

FIG. 3. Normalized acceleration gradient as a function of (a) vacuum channel width and (b) Au layer thickness. All other parameters correspond to the optimized MLA for $800 \mathrm{~nm}$ resonance.

for $d_{z}=40 \mathrm{~nm}$. This is a direct result of the field enhancement and localization properties induced by the nanoantennas. The field localization points where the field enhancement is maximal are located at the gaps of the slots and at the apertures adjacent to the dipoles, approximately at the mid thickness of the Au layer. Therefore, smaller $d_{z}$ results in stronger coupling of these intense fields to the vacuum channel, consequently increasing the acceleration gradient. However, for practical reasons, it is desired to employ the widest possible vacuum channel. Hence, a $100 \mathrm{~nm}$ width is a good compromise, as it yields a relatively large gradient while retaining a sufficiently wide vacuum channel. Note that at a width of $\lambda_{0} / 4=200 \mathrm{~nm}$ (the optimum width of the dielectric field reversal accelerator) the acceleration gradient equals $1.03 E_{0}$ (assuming equivalent laser beam power and double illumination). This value still exceeds the $0.49 E_{0}$ gradient reported in Ref. [13].

The Au thickness affects the resonance wavelength and can therefore modify the acceleration gradient as well. This effect is clearly seen in Fig. 3(b) exhibiting a distinctive peak at $t=40 \mathrm{~nm}$, for which the resonance wavelength is $800 \mathrm{~nm}$. Note that as the field enhancement is mostly determined by the $\mathrm{Au}-\mathrm{SiO}_{2}$ and $\mathrm{Au}$-Vacuum boundary surfaces it is less sensitive to thickness variations of the $\mathrm{Au}$ layer for thick Au layers.

\section{ACCELERATION GRADIENT BOUND, LOADED GRADIENT, AND BUNCH CHARGE LIMIT}

To deduce the MLA acceleration gradient bound, an investigation of the expected structural damage is required. Structural damage under laser illumination is caused mainly due to melted heat zones and laser ablation, where for an ultrashort pulse laser the latter is the dominant effect [34-36]. While some metals, such as $\mathrm{Cu}$, experience lower damage thresholds than $\mathrm{SiO}_{2}$ and other dielectrics [37,38], thin Au films on dielectric substrates can attain similar and even larger damage thresholds from that of $\mathrm{SiO}_{2}$ when exposed to femtosecond light pulses. This depends on the exact pulse characteristics, the dielectric substrate, and the fabrication surface quality $[36,39,40]$. Therefore, we assume a representative damage threshold similar to that of $\mathrm{SiO}_{2}\left(\sim 2 \mathrm{~J} / \mathrm{cm}^{2}\right.$ for a 100 fs pulse at $800 \mathrm{~nm}$ [37-39] corresponding to a peak electric field of $E_{0} \sim 10 \mathrm{GV} / \mathrm{m}$ ). To deduce the acceleration gradient bound, we investigated the MLA field magnitude distribution at resonance. Figure 4 describes the $y z$ cross section of this distribution (normalized to $E_{0}$ ), where the white lines represent the MLA contour. As can be seen, the field is localized in the gaps, between the metallic sections. Moreover, due to the mushroomlike apertures, the field in the $\mathrm{SiO}_{2}$ is relatively small. The maximal value is experienced at the surfaces between the different media where it reaches a maximal value of $5.69 E_{0}$ in the $\mathrm{Au}$ (at the patch edges) and $3.88 E_{0}$ in the $\mathrm{SiO}_{2}$ (at the mushroom edges). Note that the field drops rapidly inside the media. Consequently, we estimate the MLA maximal unloaded acceleration gradient to be $4.64 \mathrm{GV} / \mathrm{m}$ for $100 \mathrm{fs}$ pulses. When applying the shortest supported pulse (16 fs-see the following analysis), the MLA bound is increased by a factor of 2.5 up to $11.6 \mathrm{GV} / \mathrm{m}$. Note that, without the mushroom vacancies, large electric fields would form in the $\mathrm{SiO}_{2}$ substrates, leading to a substantially lower bound $(\sim 0.6 \mathrm{GV} / \mathrm{m}$ for a 100 fs pulse).

These relatively large bounds render the MLA highly power efficient. However, this may limit the amount of charge that can be accelerated due to the wakefields of the bunch charge. These wakefields alter the acceleration gradient as EM fields moving with the relativistic particles encounter geometric variations of the MLA structure, get scattered, and act on trailing particles in the same or consecutive bunch [41]. To explore this effect and deduce a bunch charge limit for the MLA structure, let us first note that the MLA supports the acceleration of only one bunch per laser pulse, as consecutive bunches which are located on different points cannot all remain synchronized with the accelerating field. As the impinging laser is perpendicular

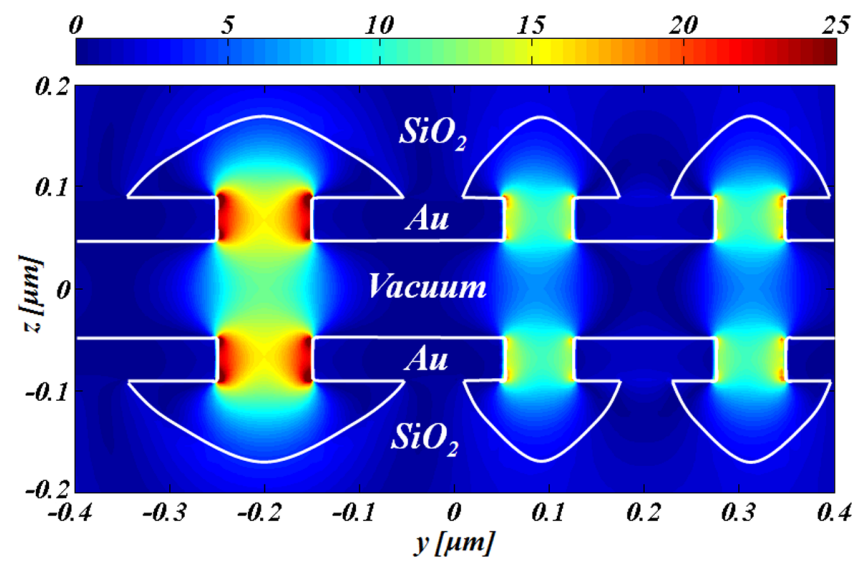

FIG. 4. A $y z$ cross section of the field magnitude distribution of the MLA structure for double illumination. The white lines represent the structural contour. 
to the particle beams, electric fields copropagating with the particle beam are negligible. Moreover, the stored EM energy in the structure is small as the structure attains a relatively small $Q$ which stems only from the nanoantenna resonance (and not from a cavity like resonance between the two MLA facets) and a small reactive impedance due to the relatively wide vacuum channel. Hence, the model that was applied for the dielectric field reversal structure [13] also applies here, with the exception that the double illumination and different acceleration gradient should be considered. Under double illumination the particle experiences a laser field of $2 E_{0}$. Therefore, in this case, by applying energy balance considerations and assuming that the effect of the nonoverlapping wakefields is small, the energy gained by a single particle passing a single unit cell can be calculated by

$$
q V=-\frac{A \cdot \lambda_{0}}{Z_{0} \cdot c}\left(E_{w}^{2}+2 E_{0} E_{w}\right),
$$

where $E_{w}$ is the wakefield of the particle which overlaps the applied laser fields and $2 E_{0}$ is the laser field which the particle experience. $Z_{0}=377 \Omega$ is the vacuum impedance and $A=D \cdot \lambda_{0}$ is the area (in the $x y$ plane) which is illuminated by the field, where $D$ is the width of the impinging pulse in the $x$ direction. The overlapping wakefield component of the particle, $E_{w}$, is by definition proportional to the laser field $E_{0}$ [13]. By assuming that the corresponding proportion factor $\alpha$ is much smaller than unity, it can be described in our case by

$$
\alpha=\frac{q Z_{S} c}{2 \lambda_{0}^{2} G},
$$

where $G$ is the unloaded gradient, $q$ is the particle charge, and $Z_{S}$ is the characteristic impedance of the structure. $Z_{S}$ is defined as the square of the voltage gained over the power lost in the structure [41]; therefore, by assuming small nanoantennas losses [24], it can be described by

$$
Z_{S}=\frac{\langle G\rangle^{2} \lambda_{0}}{D} Z_{0}
$$

where $\langle G\rangle$ is the normalized acceleration gradient which corresponds to 2.64 in the MLA. Taking $D$ to be equal to $10 d_{x}=5 \mu \mathrm{m}$, which is sufficiently wide to attain the required accelerating field profile, the characteristic impedance of the MLA equals $420.4 \Omega$, larger than the $37 \Omega$ impedance of the dielectric field reversal structure [13] due to the larger acceleration gradient and the double illumination. Consequently, $\alpha$ (for the $100 \mathrm{fs}$ pulse) is equal to $3.4 \times 10^{-6}$, thus consistent with our assumption. The overlapping wakefield of a bunch with $N_{b}$ particles is proportional to the total bunch charge $\left(N_{b} q\right)$, where it consists of a coherence contribution of the single particles wakefields [13]. Following, the loaded gradient of the bunch can be described in the MLA case under double illumination by

$$
G_{L}=G-\frac{N_{b} q c}{4 \lambda_{0}^{2}} Z_{S}
$$

In addition, the efficiency $\eta$ is defined as the ratio between the energy gained by the accelerated bunch and the applied laser energy. Therefore, without loss of generality, for a unit cell length of $\lambda_{0}$ and corresponding time $\lambda_{0} / c$ during which the bunch is affected by the pulse in this unit cell, the efficiency is related to the bunch charge as follows:

$$
\eta=\frac{N_{b} q c G_{L}}{P_{L}}
$$

where $P_{L}=\frac{A E_{0}^{2}}{2 Z_{0}} \cdot 2$ is the applied laser power that is required for a double illumination of amplitude $E_{0}$. By introducing Eq. (7) into Eq. (8), it can be seen that there is a quadratic relation between $\eta$ and $N_{b}$. Hence $\eta$ has an optimum which can be derived by deducing the extremum of this relation. At this optimum the loaded gradient corresponds to $G_{L}=0.5 G$ and the charge limit equals

$$
N_{b_{-} \mathrm{opt}}=\frac{2 G \lambda_{0}^{2}}{q c Z_{s}} .
$$

At the bound acceleration gradient for $100 \mathrm{fs}$, the charge limit is thus $N_{b o p t} \sim 2.94 \times 10^{5}$ (assuming electron acceleration). This charge limit is significantly larger than the charge per bunch supported by waveguide-based geometries [9]. However, it is small compared to RF-technologybased accelerators. Nevertheless, high particle beam luminosities can be yet attained by applying small beam diameters and working with high bunch and laser repetition rates [42]. Note that the MLA charge limit is approximately an order of magnitude smaller than the dielectric field reversal bunch charge limit $\left(\sim 3 \times 10^{6}\right)$. This is due to its larger acceleration gradient and relatively large characteristic impedance.

\section{TEMPORAL LIMITATIONS AND SHORTEST SUPPORTED PULSE}

The effective bandwidth of the MLA determines its compatibility to ultrashort pulse laser operation. The passband of the MLA stems from the resonance linewidth of the nanoantennas and from the accumulated desynchronization which occurs when the wavelength of the incident field differs from the longitudinal structure periodicity $\left(d_{y}=\lambda_{0}\right)$. The solid line in Fig. 5(a) depicts the calculated spectral bandwidth [full width half maximum (FWHM)] of the MLA structure detailed in Table I as a function of the number of periods. As expected, it decreases monotonically for a larger number of periods due to the desynchronization effect, where the width at $N=1$ 


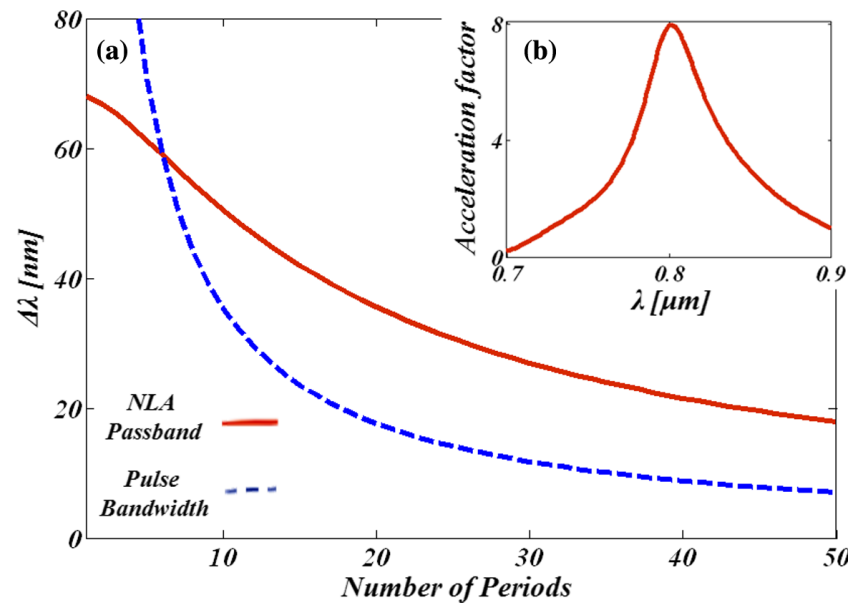

FIG. 5. (a) MLA passband (solid red line) and pulse bandwidth (dashed blue line) as a function of the MLA number of periods. (b) Normalized acceleration factor for six periods long MLA.

corresponds to that of the resonance linewidth $(68 \mathrm{~nm})$. To evaluate the shortest temporal pulse supported by the MLA structure, we compare the FWHM bandwidth of a transform-limited Gaussian temporal pulse to the passband of the MLA. Assuming a center wavelength of $\lambda_{0}$ and a $\tau_{p}$ FWHM temporal width, the pulse bandwidth is given by [43]

$$
\Delta \lambda=\frac{\lambda_{0}^{2}}{c} \cdot \frac{2 \ln (2)}{\pi} \cdot \frac{1}{\tau_{p}}
$$

The electrons passing through the MLA are accelerated only when a laser pulse exists, i.e., along a path length of $\tau_{p} c$. This length corresponds to $N_{\text {period }}=\tau_{p} \cdot c / \lambda_{0}$, the number of consecutive periods. By introducing this relation into Eq. (10), the pulse bandwidth can be described as a function of the corresponding number of structure periods:

$$
\Delta \lambda=\frac{2 \ln (2)}{\pi} \cdot \frac{\lambda_{0}}{N_{\text {period }}} .
$$

The dashed line in Fig. 5(a) describes the pulse bandwidth as a function of the number of periods that it occupies. As can be seen, this bandwidth also decreases monotonically with an increasing number of periods. Nonetheless, when the pulse occupies more than six periods (a pulse larger than $16 \mathrm{fs}$ ), the passband of the MLA becomes larger than the pulse bandwidth. Therefore, we conclude that the MLA supports excitation pulses which are longer than $16 \mathrm{fs}$. Note that continues wave operation is also supported by the structure; however, in this case it seems that high $Q$ structures may be preferable. The total normalized acceleration factor, i.e., $G N_{\text {period }} / 2 E_{0}$, corresponding to the crossing point is depicted in Fig. 5(b). As can be seen, the response is almost symmetrical, centered at the resonance wavelength, $\lambda_{0}=800 \mathrm{~nm}$.

\section{CONCLUSIONS}

The MLA structure proposed here constitutes a new paradigm for laser-driven particle acceleration. It utilizes the strong field enhancement and localization provided by a metasurface comprising a periodic slot-patch metallic nanoantenna array to attain net acceleration. This approach yields a relatively large acceleration gradient while supporting ultrashort laser operation. The proposed MLA design was optimized for relativistic particle beams and laser operation at $\lambda=800 \mathrm{~nm}$. Under symmetrical illumination, it provides an acceleration gradient of $G=2.64 E_{0}$, which is 3.81 times larger than the expected maximal acceleration gradient of the dielectric field reversal structure. The symmetric illumination contributes a factor of $\sqrt{2}$ to this ratio. Elaborating on this observation, an MLA enabling illumination from all four facets of the vacuum channel can double the efficiency, and an MLA with cylindrical symmetry which utilizes radial illumination seems the most efficient. Nevertheless, these structures are more complicated to realize. The presented structure is applicable for ultrashort laser operation up to a lower bound of $16 \mathrm{fs}$. The maximal acceleration gradient at this bound is estimated as $11.6 \mathrm{GV} / \mathrm{m}$. This estimation is based on empirical damage thresholds and on calculations of the maximal field magnitude in the media. The latter values were reached by the introduction of the mushroomlike vacancies which reduce the maximal field in the substrates. We believe that the proposed MLA opens new research directions for additional plasmonic-based accelerators. Particularly, the large number of structural degrees of freedom facilitates the design of MLAs operating at different laser center wavelengths and for slower particle beams. The main challenge in designing these structures is attaining a field profile that resembles the required spatial harmonic as slower particle acceleration necessitates smaller unit cells. The MLA design versatility, along with the efficient ultrashort pulse operation and the relatively high acceleration gradient, renders the MLA a promising concept for future laser-driven particle accelerators operating at the ultrashort pulse regime.

\section{ACKNOWLEDGMENTS}

The authors thank A. Gover for thorough and stimulating discussions regarding the MLA concept and its analysis. This research was supported by the Tel-Aviv University Nanotechnology center graduate student fellowship.

[1] C. Joshi, IEEE Trans. Nucl. Sci. 32, 1576 (1985)

[2] J. Rosenzweig, A. Murokh, and C. Pellegrini, Phys. Rev. Lett. 74, 2467 (1995).

[3] C. E. Clayton, K. A. Marsh, A. Dyson, M. Everett, A. Lal, W. P. Leemans, R. Williams, and C. Joshi, Phys. Rev. Lett. 70, 37 (1993). 
[4] P. Sprangle, E. Esarey, A. Ting, and G. Joyce, Appl. Phys. Lett. 53, 2146 (1988).

[5] I. Blumenfeld et al., Nature (London) 445, 741 (2007).

[6] V. Malka, J. Faure, Y. A. Gauduel, E. Lefebvre, A. Rousse, and K. T. Phuoc, Nat. Phys. 4, 447 (2008).

[7] R. Palmer et al., in Laser Acceleration of Particles, edited by C. Joshi and T. Katsouleas, AIP Conf. Proc. Vol. 130 (AIP, New York, 1985), pp. 234-252.

[8] J. R. Fontana and R. H. Pantell, J. Appl. Phys. 54, 4285 (1983).

[9] B. Cowan, Phys. Rev. ST Accel. Beams 11, 011301 (2008).

[10] Y. C. Huang, D. Zheng, W. M. Tulloch, and R. L. Byer, Appl. Phys. Lett. 68, 753 (1996).

[11] R. B. Yoder and J. B. Rosenzweig, Phys. Rev. ST Accel. Beams 8, 111301 (2005).

[12] B. Naranjo, A. Valloni, S. Putterman, and J. B. Rosenzweig, Phys. Rev. Lett. 109, 164803 (2012).

[13] T. Plettner, P. P. Lu, and R. L. Byer, Phys. Rev. ST Accel. Beams 9, 111301 (2006).

[14] E. A. Peralta et al., Nature (London) 503, 91 (2013).

[15] S. A. Maier, Plasmonics: Fundamentals and Applications (Springer, New York, 2007).

[16] L. Novotny and N. F. van Hulst, Nat. Photonics 5, 83 (2011).

[17] L. Novotny and B. Hecht, Principles of Nano-Optics (Cambridge University Press, Cambridge, England, 2006).

[18] K. Li, M. I. Stockman, and D. J. Bergman, Phys. Rev. Lett. 91, 227402 (2003).

[19] P. J. Schuck, D. P. Fromm, A. Sundaramurthy, G. S. Kino, and W. E. Moerner, Phys. Rev. Lett. 94, 017402 (2005).

[20] S. Lal, S. Link, and N. J. Halas, Nat. Photonics 1, 641 (2007).

[21] J. Scheuer, Opt. Express 19, 25454 (2011).

[22] A. Bouhelier, M. Beversluis, A. Hartschuh, and L. Novotny, Phys. Rev. Lett. 90, 013903 (2003).

[23] S. Kim, J. Jin, Y.-J. Kim, I.-Y. Park, Y. Kim, and S.-W. Kim, Nature (London) 453, 757 (2008).

[24] Y. Yifat, Z. Iluz, M. Eitan, I. Friedler, Y. Hanein, A. Boag, and J. Scheuer, Appl. Phys. Lett. 100, 111113 (2012).
[25] L. Novotny, Phys. Rev. Lett. 98, 266802 (2007).

[26] K. D. Ko, A. Kumar, K. H. Fung, R. Ambekar, G. L. Liu, N. X. Fang, and K. C. Toussaint, Nano Lett. 11, 61 (2011).

[27] A. Alù and N. Engheta, Phys. Rev. Lett. 101, 043901 (2008).

[28] Y. Yifat, Z. Iluz, D. Bar-Lev, M. Eitan, Y. Hanein, A. Boag, and J. Scheuer, Opt. Lett. 38, 205 (2013).

[29] D. Bar-Lev and J. Scheuer, Opt. Express 21, 29165 (2013).

[30] N. Yu, P. Genevet, F. Aieta, M. A. Kats, R. Blanchard, G. Aoust, J-P Tetienne, Z. Gaburro, and F. Capasso, IEEE J. Sel. Top. Quantum Electron. 19, 4700423 (2013).

[31] Y. Yifat, M. Eitan, Z. Iluz, Y. Hanein, A. Boag, and J. Scheuer, Nano Lett. 14, 2485 (2014).

[32] FDTD Solutions, Release 8.6.4, http://www.lumerical .com.

[33] E. D. Palik, Handbook of Optical Constants of Solids (Academic, New York, 1985).

[34] B. C. Stuart, M. D. Feit, S. Herman, A. M. Rubenchik, B. W. Shore, and M. D. Perry, Phys. Rev. B 53, 1749 (1996).

[35] K. Venkatakrishnan, B. Tan, and N. R. Sivakumar, Opt. Laser Technol. 34, 575 (2002).

[36] K. Venkatakrishnan, P. Stanley, N.R. Sivakumar, B. Tan, and L. E. N. Lim, Appl. Phys. A 77, 655 (2003).

[37] K. Soong, R. L. Byer, E. R. Colby, R. J. England, and E. A. Peralta, AIP Conf. Proc. 1507, 511 (2012).

[38] D. Du, X. Liu, G. Korn, J. Squier, and G. Mourou, Appl. Phys. Lett. 64, 3071 (1994).

[39] B. C. Stuart, M. D. Feit, S. Herman, A. M. Rubenchik, B. W. Shore, and M. D. Perry, J. Opt. Soc. Am. B 13, 459 (1996).

[40] B. Tan, A. Dalili, and K. Venkatakrishnan, J. Appl. Phys. A 95, 537 (2009).

[41] T. P. Wangler, Principles of RF Linear Accelerators (Wiley, New York, 1998).

[42] R. H. Siemann, Phys. Rev. ST Accel. Beams 7, 061303 (2004).

[43] A. M. Weiner, Ultrafast Optics (Wiley, New York, 2009). 